\title{
Kinematics of Trunk-Like Robots with Piezo Actuators
}

\author{
Aistis AUGUSTAITIS*, Vytautas JURE்NAS**, Genadijus KULVIETIS*** \\ *Vilnius Gediminas Technical University, Sauletekio 11, 10223 Vilnius, Lithuania, E-mail: aistis.augustaitis@vgtu.lt \\ **Kaunas University of Technology, K. Donelaičio g. 73, 44249 Kaunas, Lithuania, E-mail: vytautas.jurenas@ktu.lt \\ ***Vilnius Gediminas Technical University, Saulètekio 11, 10223 Vilnius, Lithuania, E-mail: genadijus.kulvietis@vgtu.lt \\ cross $^{\text {ref }}$ http://dx.doi.org/10.5755/j01.mech.24.2.19205
}

\section{Introduction}

It is important that astronautic equipment should be light and compact as possible, but strong enough to withstand heavy payloads or shocks and the influence of temperature differences. The same can be said about astronautic manipulator. It should have many degrees of freedom and a big length when extended also [1].

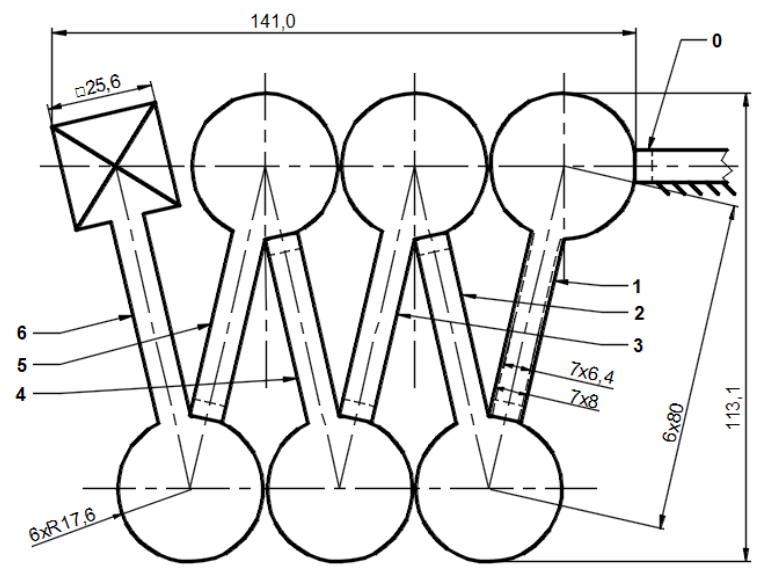

Fig. 1 Tightened trunk-like robot with piezo actuators

Hyper-redundant robots (HRR) have many more degrees of freedom (DOFs) than required in 2D or 3D environments. This feature enables them to handle more constraints, those can be found in highly convoluted volumes. Despite the advantage of high dexterity level, we have to provide its major challenges: mechanism design, control and path planning [2].

A structure scheme of a trunk-like robot (HRR) is shown in figure 1 , which is controlled by spherical piezoelectric actuators. HRR can also be called as a snake-like robot. Piezo actuator is an ultrasonic device, which can be explained by the change of motion occasion. These occasions are frictional and inertial effects when piezoelectric element generates micro-oscillations. The piezo robot has 6 movable links and 18 DOFs for angular movements, excluding a gripper. An ordinary movable link (No. 1-5) is consisted of hollow ball and cylindrical tube with modified piezo transducer at its end. The first link-tube (No. 0) with piezo transducer is fixed and can induce the motion of the first movable link (No. 1). Permanent magnets or electromagnets should be used to ensure the flexible connections between serially connected links. Vacuum pressures inside piezo tubes can be generated to ensure higher braking moments between connected links. This option can be used to lock the joints with passive links, because wide contact area and high value of friction coefficient can be achieved. A hollow ball can be consisted of layers with different materials to ensure lightness, high stiffness and good magnetic features. Plastic rings between the main bodies of ordinary movable links should be used to eliminate the disturbance from one actuator to another. In order to reduce the ratio of hollow ball and cylindrical tube diameters and to ensure the HRR ability to be compact the centerline of almost every tube should be changed to curve (Fig. 2). The configuration in figure 2 can be considered as primary.

Piezo robots are suitable for astronautics because of these inherent advantages: high accuracy, short response time, simple mechanical design and low weight [3-5].

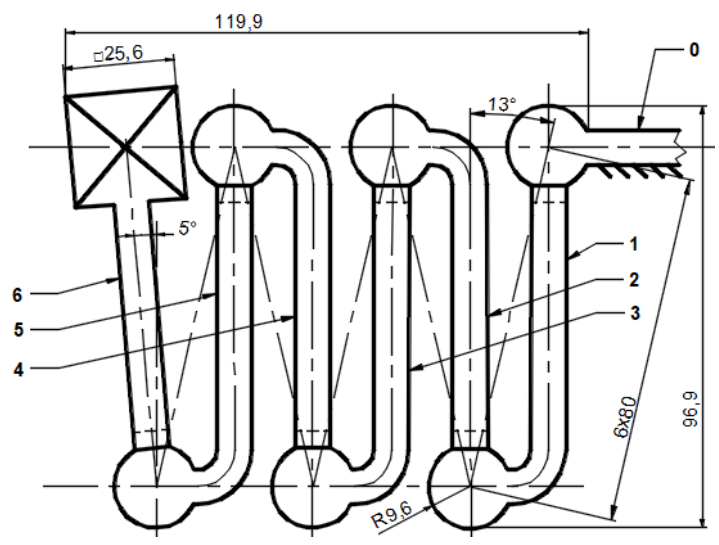

Fig. 2 Tightened trunk-like robot with curved links

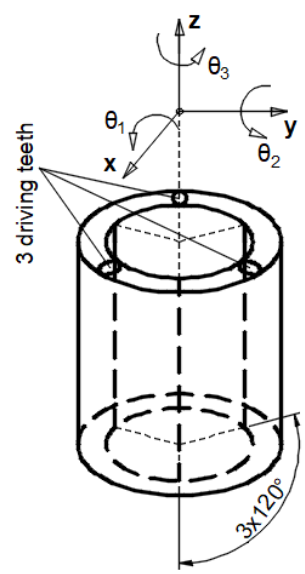

Fig. 3 Structure of modified piezo tube

A piezo transducer is chosen to be a shape of tube also. The casual structure of piezo tube has to be modified in order to establish the ability to rotate independently about three perpendicular axes (Fig. 3). According to this, inside electrode of the tube are divided into three separate electrodes. The shape and area of mentioned electrodes should 
be identical. Driving teeth are used to ensure correct contact zones with the ball. Control techniques for multi-axis piezo actuators are already defined [6].

The first part of the article introduces the structure and direct kinematics for trunk-like robot with piezo actuators (Sections 1-2), which can be employed in astronautics. The final part of the work is made to show the methods of inverse kinematics (Section 3) and to present a new method of geometric approach (Section 4). A concise algorithm of proposed geometric approach with results are also shown in section 4.

\section{Review of direct kinematics}

Any complex joint can be analyzed as the system with single joints. A single joint has only one degree of freedom. The piezo robot is consisted of spherical actuators and serial links with permanent axes directions in accordance to their orientations (Figs. 4-5). One of the possible configurations of tightened robot (Fig. 1) is shown in Fig. 5, when only rotations about $x$ axes have been used (Table 1).

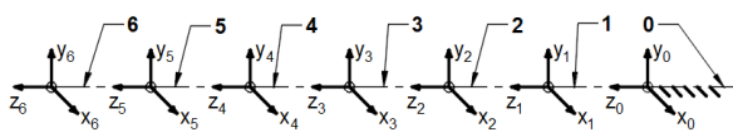

Fig. 4 Kinematic scheme of the extended robot

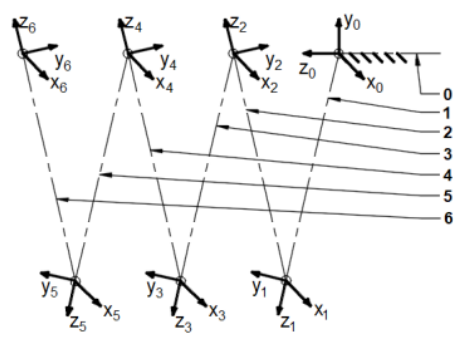

Table 1

Values of joints angles at the tightened position

\begin{tabular}{|c|c|c|c|c|}
\hline $\begin{array}{c}\text { Joint } \\
\text { no. }\end{array}$ & $\begin{array}{c}\text { Between } \\
\text { links }\end{array}$ & $\Theta_{x}\left(^{\circ}\right)$ & $\Theta_{y}\left(^{\circ}\right)$ & $\Theta_{z}\left(^{\circ}\right)$ \\
\hline 1 & $0-1$ & 77,0 & 0 & 0 \\
\hline 2 & $1-2$ & $-154,0$ & 0 & 0 \\
\hline 3 & $2-3$ & 154,0 & 0 & 0 \\
\hline 4 & $3-4$ & $-154,0$ & 0 & 0 \\
\hline 5 & $4-5$ & 154,0 & 0 & 0 \\
\hline 6 & $5-6$ & $-154,0$ & 0 & 0 \\
\hline
\end{tabular}

According to the extraordinary motion of the ball using permanent-fixed axes, it is chosen to use roll-pitchyaw (RPY) representation [7, 8] to perform the transformation of coordinate frames. Single joint $i$ has only one variable $\theta_{i}$. To perform the kinematic analysis, we can rigidly attach a local coordinate frame to the kinematic end of each real link. The $z$ axis of link frame is coincident with its longitudinal axis. The frame $x_{0} y_{0} z_{0}$ is attached to the kinematic origin of the first movable link and can be taken as inertial frame [7]. Let we consider, that at the beginning the axes of all coordinate frames of trunk robot were made coincident respectively (Fig. 4).

The positive sense for angles is determined by the right-hand rule [7]. At first let we determine the orientation matrix of the first link, when the sequence of rotation axes is $x_{0}-y_{0}-z_{0}$ (Eq. (1)). Then we can evaluate the transformation matrix Eq. (2) between 1 and 0 coordinate frames using the transposition matrix of the first link $T\left(r_{1}\right)$. Similarly, we can determine the transformation matrix of the second link or other. The transposition matrix $T\left(r_{i}\right)$ is the sum of the unit matrix $(4 \times 4)$ and the position matrix of the $i$-th link kinematic line before a rotation. The transformation matrix between 6 and 0 coordinate frames can be determined using the Eq. (3) [7].

Fig. 5 Kinematic scheme of the tightened robot

$$
\begin{gathered}
R_{1}^{0}\left(\theta_{1}, \theta_{2}, \theta_{3}\right)=R_{z}\left(\theta_{3}\right) \cdot R_{y}\left(\theta_{2}\right) \cdot R_{x}\left(\theta_{1}\right)=\left[\begin{array}{cccc}
c_{3} c_{2} & c_{3} s_{2} s_{1}-s_{3} c_{1} & c_{3} s_{2} c_{1}+s_{3} s_{1} & 0 \\
s_{3} c_{2} & s_{3} s_{2} s_{1}+c_{3} c_{1} & s_{3} s_{2} c_{1}-c_{3} s_{1} & 0 \\
-s_{2} & c_{2} s_{1} & c_{2} c_{1} & 0 \\
0 & 0 & 0 & 1
\end{array}\right], \\
M_{1}^{0}=R_{1}^{0} T\left(r_{1}\right)=R_{1}^{0}\left[\begin{array}{llll}
1 & 0 & 0 & 0 \\
0 & 1 & 0 & 0 \\
0 & 0 & 1 & r_{1} \\
0 & 0 & 0 & 1
\end{array}\right]=\left[\begin{array}{cccc}
c_{3} c_{2} & c_{3} s_{2} s_{1}-s_{3} c_{1} & c_{3} s_{2} c_{1}+s_{3} s_{1} & r_{1}\left(c_{3} s_{2} c_{1}+s_{3} s_{1}\right) \\
s_{3} c_{2} & s_{3} s_{2} s_{1}+c_{3} c_{1} & s_{3} s_{2} c_{1}-c_{3} s_{1} & r_{1}\left(s_{3} s_{2} c_{1}-c_{3} s_{1}\right) \\
-s_{2} & c_{2} s_{1} & c_{2} c_{1} & r_{1}\left(c_{2} c_{1}\right) \\
0 & 0 & 0 & 1
\end{array}\right],
\end{gathered}
$$

$M_{6}^{0}=M_{1}^{0} M_{2}^{1} M_{3}^{2} M_{4}^{3} M_{5}^{4} M_{6}^{5}$

where $1,2,3$ are $x_{0}, y_{0}$ and $z_{0}$ axes; $c_{1}-\cos \left(\theta_{1}\right) ; s_{1}-\sin \left(\theta_{1}\right)$ and so on; $r_{i}$ is the length of $i$-th link kinematic line.

\section{Methods of inverse kinematics}

The resolved-motion (RM) method can be used to solve inverse kinematics (IK) for various design manipulators. This method has some weak points $[9,10]$ :

- It has intrinsic inaccuracy because of linear approximation characteristics of the Jacobian matrix. Thus it accumulates errors which become larger as the velocity increases.

- It does not directly give the joint values for a given location of the end effector.

The closed-form solution formula of IK for manipulator with redundancy can be derived using the Lagrangian multiplier method. The method is more efficient and accurate in solving given trajectories than the RM method. The solution of the inverse kinematics can be obtained using a nonlinear optimization algorithm, which uses a modified Newton-Raphson technique for solving nonlinear equations [9]. 
The algorithm of the IK can be based on a dynamic reformulation of the problem leading to a closed-loop scheme. Its stability is assured by choosing a control, which only involves direct computation of manipulator kinematics. An obstacle avoidance and limited joint range can be evaluated using two last methods. Vector formulations for solution of IK problem were developed and led to efficient computer algorithms. To overcome the difficulties encountered near kinematic singularities, the method of change the exact inverse problem to a damped-least squares problem was proposed. This method was not confirmed [9].

Many methods of inverse kinematics are based on evaluating joints velocities. This approach is obtained using Jacobian or pseudoinverse matrices, those are ill-conditioned near kinematic singularities $[9,10]$. It is important to rationally use the extra DOFs to achieve objectives such as an avoidance of singularity or an obstacle for mentioned manipulators.

There are only iterative solutions for IK of various structure robots using direct mapping that can be computationally expensive. However, this method is attractive because it eliminates the essential problems of the RM and similar methods. Relatively little research is made in the development of the mentioned method for kinematically redundant manipulators [9].

\section{Theory of inverse kinematics for the robot with two different spherical actuators using a geometric ap- proach}

Using Fig. 6 can be established that are two different actions of real spherical actuators. This time the first link has two piezoelectric transducers at its ends. The second link acts the same as every movable link in Fig. 1. The second spherical actuator (Fig. 6) can be assumed to perform a direct action and the other - opposite or inverse action. Direct action could be a movement when the zero coordinate frame, which belongs to the hemisphere, moves in accordance to the first coordinate frame, which introduces the position of piezotube at the beginning of the first link. The last statement is a false example, because the zero frame is inertial and the real action is inverse.

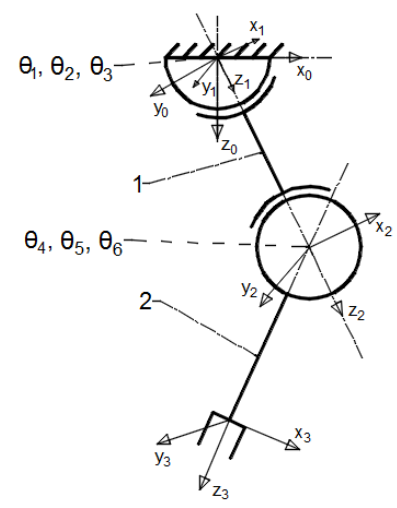

Fig. 6 Piezoelectric robot with two different spherical actuators

If we consider that the beginning of finite rod is attached to spherical joint, then only two single joints are enough to estimate the end position of the rod in a spherical surface. If the length of the rod is fixed, then we can freely change only two projections from three and write only two independent equations for estimation of variables.

The orientation of robot final link about its longitudinal axis can be important, but chosen algorithm below ignores it. This feature is useless, if the robot has a nondirectional end-effector like a magnetic, vacuum or mechanical (with three or more fingers) gripper. If the final spherical actuator of manipulator has an inverse action, then its rotation about $z$ axis has no meaning to the global coordinates of the end-effector and can be used freely. To establish the accurate orientation of robot final link some additional calculations should be included to mentioned algorithm.

The first step of the algorithm is to determinate the global coordinates of the kinematic ends of manipulator links and to estimate the global projections of the links. Geometric approach is a direct way-algorithm to avoid obstacles in workspace. The constraints of robot joints can be included into mentioned algorithm and additional tests for that may be omitted. If desired coordinates of robot end-effector can't be reached, then any solution can be found. The algorithms for estimation the global coordinates of the kinematic ends are already defined in other articles $[9,10]$.

Here will be used a transformation algorithm using two local transposition vectors $(4 \times 1)$ of the same link. Firstly, we consider a spherical actuator $i-l$ with a direct action. If we know the projection values of the final transposition vector $T_{i(F)}^{i-1}$ of the $i$-th link and the values of the desired transposition vector $T_{i(D)}^{i-1}$, then the equation 4 can be written for the transformation using the sequence of rotation axes $x$ $y$. The correction angle of $x$ axis has an index 1 and the correction angle of $y$ axis -2 all the time. The explicit form of the expression 4 is given in the equation 5 . If the equation 6 , which can be found from the last equation, does not have a solution, then the sequence of rotational axes has to be changed into $y$ - $x$. In accordance to that we can write the equation 7. The explicit form of the last expression is given in the equation 8 .

If the function with range $\left[-r_{i}^{\prime}, r_{i}^{\prime}\right]$ in the Eq. (6) has no solution (Fig. 7), then we can write an important condition Eq. (9). In accordance to the fact, that each link has a fixed length $r_{i}$, we can write the Eqs. (10) and (11). The condition for the solution existence of the Eq. (8) can be written Eq. (12). The maximum modulus of $x_{i(D)}^{i-1}$ can be evaluated using the Eq. (13) and the modulus of $x_{i(F)}^{i-1}$ can be evaluated using the Eq. (14).

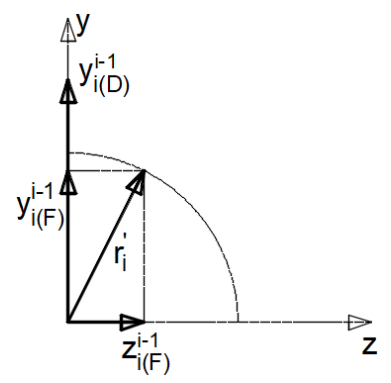

Fig. 7 The change of link projections during rotation

If the condition in the Eq. (9) is true, then the Eq. (12) is also true (using the Eqs. (13) and (14)). This means, 
that the Eq. (8) has a solution, when the Eq. (5) has not. The opposite statement can be proved using the same way.

$$
\begin{aligned}
& R_{c o r_{-} 1}^{d i r_{i(F)}} T_{y}^{i-1}=R_{y}\left(\theta_{2}\right) R_{x}\left(\theta_{1}\right) T_{i(F)}^{i-1}=T_{i(D)}^{i-1} . \\
& {\left[\begin{array}{cccc}
c_{2} & s_{1} s_{2} & c_{1} s_{2} & 0 \\
0 & c_{1} & -s_{1} & 0 \\
-s_{2} & c_{2} s_{1} & c_{1} c_{2} & 0 \\
0 & 0 & 0 & 1
\end{array}\right]\left\{\begin{array}{c}
x_{i(F)}^{i-1} \\
y_{i(F)}^{i-1} \\
z_{i(F)}^{i-1} \\
1
\end{array}\right\}=\left\{\begin{array}{c}
x_{i(D)}^{i-1} \\
y_{i(D)}^{i-1} \\
z_{i(D)}^{i-1} \\
1
\end{array}\right\} .} \\
& c_{1} y_{i(F)}^{i-1}-s_{1} z_{i(F)}^{i-1}=y_{i(D)}^{i-1} . \\
& R_{c o r_{-} 2}^{d i r} T_{i(F)}^{i-1}=R_{x}\left(\theta_{1}\right) R_{y}\left(\theta_{2}\right) T_{i(F)}^{i-1}=T_{i(D)}^{i-1} . \\
& {\left[\begin{array}{cccc}
c_{2} & 0 & s_{2} & 0 \\
s_{1} s_{2} & c_{1} & -c_{2} s_{1} & 0 \\
-c_{1} s_{2} & s_{1} & c_{1} c_{2} & 0 \\
0 & 0 & 0 & 1
\end{array}\right]\left\{\begin{array}{c}
x_{i(F)}^{i-1} \\
y_{i(F)}^{i-1} \\
z_{i(F)}^{i-1} \\
1
\end{array}\right\}=\left\{\begin{array}{c}
x_{i(D)}^{i-1} \\
y_{i(D)}^{i-1} \\
z_{i(D)}^{i-1} \\
1
\end{array}\right\} .} \\
& \sqrt{\left(y_{i(F)}^{i-1}\right)^{2}+\left(z_{i(F)}^{i-1}\right)^{2}}=\left|r_{i}^{\prime}\right|<\left|y_{i(D)}^{i-1}\right| . \\
& \left(x_{i(F)}^{i-1}\right)^{2}+\left(y_{i(F)}^{i-1}\right)^{2}+\left(z_{i(F)}^{i-1}\right)^{2}=\left(r_{i}\right)^{2} . \\
& \left(x_{i(D)}^{i-1}\right)^{2}+\left(y_{i(D)}^{i-1}\right)^{2}+\left(z_{i(D)}^{i-1}\right)^{2}=\left(r_{i}\right)^{2} . \\
& \sqrt{\left(x_{i(F)}^{i-1}\right)^{2}+\left(z_{i(F)}^{i-1}\right)^{2}} \geq\left|x_{i(D)}^{i-1}\right| . \\
& \left|x_{i(D)}^{i-1}\right|_{\max }=\sqrt{\left(r_{i}\right)^{2}-\left(y_{i(D)}^{i-1}\right)^{2}} . \\
& \left|x_{i(F)}^{i-1}\right|=\sqrt{\left(r_{i}\right)^{2}-\left(y_{i(F)}^{i-1}\right)^{2}-\left(z_{i(F)}^{i-1}\right)^{2}} .
\end{aligned}
$$

Now we can consider a spherical actuator $k-1$ with an inverse action. The orientation of the $k$-th link is controlled by the last actuator. This time only orientation matrices of the $k$-th link are most important. The final orientation matrix is marked as $F_{k}$ Eq. (15) and the desired orientation matrix $-D_{k}$ Eq. (16). If the sequence of rotation axes is $x-y$ and the values of the matrices are known, then we can write the Eq. (17). An orientation matrix is orthogonal. This means, that the meanings of inverse and transpose orthogonal matrices are the same. Using explicit form of the last equation we can derive three Eqs. (18)-(20) in accordance to the link ( $z$ axis) projections. If $c_{1} c_{2}$ is taken as variable $x$, $s_{1} c_{2}-y, s_{2}-z$, then we get the system of linear equations. The solution for variable $x$ is given in the Eq. (21), the solution for variable $y$ - in the Eq. (22), the solution for variable $z$ - in the Eq. (23). The value of $D_{3 \_k}$ can be derived using the Eq. (24), the value of $D_{6 \_k}-$ the Eq. (25) and the value of $D_{9 \_k}-$ the Eq. (26).

$$
\begin{gathered}
F_{k}=\left[\begin{array}{cccc}
F_{1_{-} k} & F_{2_{-} k} & F_{3_{-} k} & 0 \\
F_{4_{-} k} & F_{5_{-} k} & F_{6_{-} k} & 0 \\
F_{7_{-} k} & F_{8_{-} k} & F_{9_{-} k} & 0 \\
0 & 0 & 0 & 1
\end{array}\right] . \\
D_{k}=\left[\begin{array}{cccc}
D_{1_{-} k} & D_{2_{-} k} & D_{3_{-} k} & 0 \\
D_{4_{-} k} & D_{5_{-} k} & D_{6_{-} k} & 0 \\
D_{7_{-} k} & D_{8_{-} k} & D_{9_{-} k} & 0 \\
0 & 0 & 0 & 1
\end{array}\right] . \\
F_{k} \cdot\left(R_{x_{-} y}^{d i r}\right)^{-1}-D_{k}=F_{k} \cdot R_{x}\left(\theta_{1}\right)^{T} \cdot R_{y}\left(\theta_{2}\right)^{T}-D_{k}=0 \\
F_{3_{-} k} c_{1} c_{2}+F_{2_{-} k} s_{1} c_{2}-F_{1_{-} k} s_{2}-D_{3_{-} k}=0 . \\
F_{6_{-} k} c_{1} c_{2}+F_{5_{-} k} s_{1} c_{2}-F_{4_{-} k} s_{2}-D_{6_{-} k}=0 . \\
F_{9_{-} k} c_{1} c_{2}+F_{8_{-} k} s_{1} c_{2}-F_{7_{-} k} s_{2}-D_{9_{-} k}=0 .
\end{gathered}
$$

$$
y=\frac{D_{6_{-} k}-D_{3_{-} k} F_{4_{-} k} / F_{1_{-} k}-x\left(F_{6_{-} k}-F_{4_{-} k} F_{3_{-} k} / F_{1_{-} k}\right)}{F_{5_{-} k}-F_{4_{-} k} F_{2_{-} k} / F_{1_{-} k}} .
$$

where $x_{k}$ is the $x$ global projection of the $k$-tk link and so on.
The relation between global $T_{j}^{0}$ and primary $T_{j}^{p r}$ transposition vectors (TVs) of the $j$-th link is given in the Eq. (27). The final TV of the $j$-th link can be defined using the Eq. (28). The desired TV of the $j$-th link can be defined using the Eq. (29).

$$
\begin{aligned}
& T_{j}^{0}=R_{1}^{0} \ldots R_{j}^{j-1} T_{j}^{p r} . \\
& T_{j(F)}^{j-1}=R_{j}^{j-1} T_{j}^{p r} . \\
& T_{j(D)}^{j-1}=\left[R_{1}^{0} \ldots R_{j-1}^{j-2}\right]^{-1} T_{j}^{0}=\left[R_{j-1}^{0}\right]^{-1} T_{j}^{0} .
\end{aligned}
$$


Finally, we can write a concise algorithm for solution of inverse kinematics for the robot. It is the sequence of actions for obtaining the correction angles from the previous configuration of the robot to the next configuration. The solution for the robot with $n$ links can have a form of matrix $(n \times 3)$. The first two columns are for the angles about $x$ and $y$ axes and the last column is for the indexes of the axes sequences. It is important to pay attention to the axes sequences for making a proper control.

STEP I. To estimate the global projections $\left(T_{1}^{0}, T_{2}^{0}\right.$ $\left., \ldots, T_{n}^{0}\right)$ of the links for desired robot configuration $[9,10]$.

STEP II. $R_{i}^{i-1}$ is equal to the unit (identity) matrix $(4 \times 4)$ at the time when all axes of the robot frames are parallel respectively. Where $i \in Z$ and $1 \leq i \leq n . R_{0}^{0}$ is equal to the unit matrix $(4 \times 4)$ all the time. $T_{i}{ }^{p r}=\left[\begin{array}{llll}0 & 0 & r_{i} & 1\end{array}\right]^{T}$, this condition is constant too.

From $i=1$ to $n \quad(n$ cycles $)$

1) $T_{i(F)}^{i-1}=R_{i}^{i-1} T_{i}^{p r}$;

2) $T_{i(D)}^{i-1}=\left[R_{i-1}^{0}\right]^{-1} T_{i}^{0}$;

3) to define which kind of action has a spherical actuator, which is attached to the beginning of the $i$-th link; 4) to determine rotation angles about $x$ and $y$ axes and the sequence of mentioned axes;

5) $R_{i}^{i-1}=R_{c o r}^{d i r} R_{i}^{i-1}$ for the $i$-th link with a direct action actuator or $R_{i}^{i-1}=R_{i}^{i-1} R_{c o r}^{i n v}$ for the $i$-th link with an inverse action actuator;

End.

Results after calculation

Table 2

\begin{tabular}{|c|c|c|c|c|c|c|c|c|c|c|c|c|c|c|c|c|c|c|}
\hline \multirow[t]{3}{*}{$\begin{array}{l}\text { Ex. } \\
\text { no. }\end{array}$} & \multicolumn{6}{|c|}{$\begin{array}{c}\text { Initial input values } \\
\text { (Activation sequence: } x-y-z \text { ) }\end{array}$} & \multicolumn{6}{|c|}{$\begin{array}{l}\text { Desired global projections (equal } \\
\text { units of length) }\end{array}$} & \multicolumn{6}{|c|}{ Results (Correctional values) } \\
\hline & \multicolumn{3}{|c|}{ First link } & \multicolumn{3}{|c|}{ Second link } & \multicolumn{3}{|c|}{ First link } & \multicolumn{3}{|c|}{ Second link } & \multicolumn{3}{|c|}{ First link } & \multicolumn{3}{|c|}{ Second link } \\
\hline & $\begin{array}{l}\Theta_{x} \\
\text { (or.) }\end{array}$ & $\begin{array}{l}\Theta_{y} \\
\left({ }^{\circ}\right)\end{array}$ & $\begin{array}{l}\Theta_{z} \\
\left({ }^{\circ}\right)\end{array}$ & $\begin{array}{l}\Theta_{x} \\
\left({ }^{\circ}\right)\end{array}$ & $\begin{array}{l}\Theta_{y} \\
\left({ }^{\circ}\right)\end{array}$ & $\begin{array}{l}\Theta_{z} \\
\left({ }^{\circ}\right)\end{array}$ & $P_{x}$ & $P_{y}$ & $P_{z}$ & $P_{x}$ & $P_{y}$ & $P_{z}$ & $\begin{array}{c}S . \\
\text { no. }\end{array}$ & $\begin{array}{c}\Phi_{x} \\
(\mathrm{rad})\end{array}$ & $\begin{array}{c}\Phi_{y} \\
(\mathrm{rad})\end{array}$ & $\begin{array}{c}S . \\
\text { no. }\end{array}$ & $\begin{array}{c}\Phi_{x} \\
(\mathrm{rad})\end{array}$ & $\begin{array}{c}\Phi_{y} \\
(\mathrm{rad})\end{array}$ \\
\hline 1 & 0 & 0 & 0 & 0 & 0 & 0 & 10 & 10 & 10 & 15 & 15 & 15 & 1 & 0,785 & $-0,616$ & 1 & 3,142 & 3,142 \\
\hline 2 & 30 & 30 & 30 & 30 & 30 & 30 & 5 & 10 & 15 & 5 & 10 & 15 & 1 & 0,530 & $-0,626$ & 1 & $-0,281$ & 5,608 \\
\hline 3 & 30 & -30 & 30 & 45 & 45 & 45 & - & - & 15 & 10 & 10 & 15 & 1 & $-1,477$ & 0,140 & 2 & 1,777 & 2,135 \\
\hline 4 & 30 & -30 & 30 & 45 & 45 & 45 & 10 & - & 15 & 10 & 10 & 15 & 1 & $-0,775$ & $-0,629$ & 2 & 2,364 & 2,558 \\
\hline 5 & 30 & -30 & 30 & 45 & 45 & 45 & 10 & 10 & 15 & 10 & 10 & 15 & 1 & 0,041 & 0,042 & 1 & 2,857 & 4,164 \\
\hline 6 & 30 & -30 & 30 & 45 & 45 & 45 & 10 & 10 & 15 & $\begin{array}{c}- \\
10\end{array}$ & $\begin{array}{c}- \\
10\end{array}$ & 15 & 1 & 0,041 & 0,042 & 2 & $-1,482$ & 2,240 \\
\hline
\end{tabular}

Results (Table 2) are obtained using the algorithm. Initial input values and desired global projections are chosen freely. The sequence of rotation axes can be chosen freely too for evaluating final orientation matrices of the links.

\section{Conclusions}

The structures and kinematic schemes of trunk-like robot with spherical piezo actuators are exposed. The DOFs number of spherical actuator can be reduced from 3 to 2 with low influence to the manipulation features of the robot and in order to facilitate the evaluation of inverse kinematics. The algorithms of inverse kinematics using Jacobian matrix sometimes can cause failures. According to this, a geometric approach to solve the inverse kinematics has been used. A concise algorithm of proposed approach and results are presented.

\section{Acknowledgement}

The work was supported by the Research Council of Lithuania under the project SmartTrunk, No. MIP$084 / 2015$.

\section{References}

1. Kauderer, A. 2013. Canadarm2 and the Mobile Servicing System. Available from Internet: https://www.nasa.gov/mission_pages/station/structure/elements/mss.html.

2. Salomon, O.; Wolf, A. 2012. Inclined links hyper-re- dundant elephant trunk-like robot, Journal of Mechanisms and robotics, Vol. 4., http://dx.doi.org/10.1115/1.4007203.

3. Bansevičius, R.; Kulvietis, G.; Macerauskas, E.; Janutenaite, J.; Drukteniene, A.; Mazeika, D. 2016. Trajectory planning for stabilization system of nanosatellite, Mechanika 2016: proceedings of the 21st international scientific conference, 22-28.

4. Bansevicius, R.; Kulvietis, G.; Mazeika, D.; Drukteiniene, A.; Tumasoniene, I.; Bakanauskas, V. 2015. The synthesis of trajectories in piezoelectric attitude control devices for nanosatellites. Journal of vibration engineering \& technologies, Chennai: Krishtel eMaging Solutions Pvt. Ltd. ISSN 2321-3558, 3(3): 345-353.

5. Zhijiang, D.; Ruochong, S.; Wei, D. 2014. A piezo-actuated high-precision flexible parallel pointing mechanism: conceptual design, development, and experiments, IEEE Transactions on Robotics, 30(1): 131-137. http://dx.doi.org/10.1109/TRO.2013.2288800.

6. Bansevicius, R.; Kargaudas, V. 2011. Attitude Control of Micro-and Nanosatellites Using Multi-degree-ofFreedom Piezoelectric Actuators. Vibration Problems ICOVP 2011, 379-384, Springer.

http://doi.org/10.1007/978-94-007-2069-5_51.

7. Spong, M. W.; Hutchinson, S.; Vidyasagar, M. 2004. Robot dynamics and control. Second Edition. Available from Internet: http://smpp.northwestern.edu/savedLiterature/Spong_Textbook.pdf. 
8. Waldron, K.; Schmiedeler, J. 2008. Springer handbook of robotics, Springer, Berlin, Heidelberg, New York. Available from Internet:

https://pdfs.semanticscholar.org/5a9c/f533505dbc 85931ce35d4ba3d3a1bfbff21a.pdf

9. Wang, L. C. T.; Chen, C. C. 1991. A combined optimization method for solving the inverse kinematics problem of mechanical manipulators, IEEE journal of Transactions on Robotics and Automation 7(4): 489499.

http://doi.org/10.1109/70.86079.

10. Ahuactzin, J. M.; Gupta, K. K. 1999. The kinematic roadmap: a motion planning based global approach for inverse kinematics of redundant robots, IEEE journal of Transactions on Robotics and Automation 15(4): 653669.

http://doi.org/10.1109/70.781970.
A. Augustaitis, V. Jurènas, G. Kulvietis

\section{KINEMATICS OF TRUNK-LIKE ROBOTS WITH PIEZO ACTUATORS}

S u m m a r y

This paper presents the investigation of direct and inverse kinematics for piezo robot with spherical actuators. Two different actions of spherical piezo actuators are introduced. Several methods to solve inverse kinematics were analyzed. A geometric approach is chosen for solving the inverse kinematics, because it has a lot of advantages in accordance to other methods. A theory and a concise algorithm to find the solutions of the inverse kinematics using the global projections of robot links is presented.

Keywords: trunk-like robot, astronautics, piezo actuator, spherical joint, direct kinematics, inverse kinematics, geometric approach. 